\title{
Elevated circulating microRNA-122 is associated with obesity and insulin resistance in young adults
}

\author{
Rui Wang ${ }^{1, *}$, Jie Hong ${ }^{1, *}$, Yanan Cao ${ }^{1, *}$, Juan Shi ${ }^{1}$, Weiqiong Gu ${ }^{1}$, Guang Ning ${ }^{1,2}$, \\ Yifei Zhang' ${ }^{1}$ and Weiqing Wang ${ }^{1}$
}

${ }^{1}$ Shanghai Clinical Center for Endocrine and Metabolic Diseases, Shanghai Institute of Endocrine and Metabolic Diseases, Department of Endocrinology and Metabolism, Shanghai Key Laboratory for Endocrine Tumors and E-Institutes of Shanghai Universities, Ruijin Hospital, Shanghai Jiao Tong University School of Medicine, 197 Ruijin 2nd Road, Shanghai 200025, China and ${ }^{2}$ Laboratory for Endocrine and Metabolism, Institute of Health Sciences, Shanghai Institutes for Biological Sciences, Chinese Academy of Sciences and Shanghai Jiao Tong University School of Medicine, Shanghai 200025, China

* (R Wang, J Hong and Y Cao contributed equally to this work)

\author{
Correspondence \\ should be addressed \\ to W Wang \\ Email \\ wqingw@hotmail.com
}

\begin{abstract}
Objective: MicroRNAs (miRNAs) are involved in the regulation of adiposity, but functional studies have yielded inconclusive results. Examining the associations of circulating miRNAs levels with obesity and insulin sensitivity in humans may lead to improved insights.

Design and methods: Serum samples collected from 112 obese and control subjects ( $50.0 \%$ men) were randomly divided and combined into four pools (28 samples in each obese or control pool). The genome-wide circulating miRNA profiles were detected via microarray. Elevated miR-122 was selected and validated in individual serum samples from 123 obese (46.7\% men) and 107 control (50.0\% men) young adults. Associations between circulating miR-122 levels and parameters related to adiposity, insulin resistance, lipid profiles and hepatic enzymes were further assessed.

Results: Thirty-four miRNAs were found to be expressed differently in the sera of obese patients compared with control subjects $(P<0.001)$. Further analyses confirmed that obese patients had 3.07-fold higher circulating miR-122 levels than controls $(P<0.001)$. Serum miR-122 levels were correlated with BMI $(r=0.469)$, alanine aminotransferase $(r=0.634)$, triglycerides $(r=0.448)$, HDL-cholesterol $(r=-0.351)$ and homeostasis model assessment of insulin resistance $(r=0.401$, all $P<0.01$ ). After controlling for confounding factors, miR-122 remained as an independent risk factor for insulin resistance $(\mathrm{OR}=3.379,95 \% \mathrm{Cl}=1.141-10.007, P=0.028)$.

Conclusions: Elevated circulating miR-122 is positively associated with obesity and insulin resistance in young adults. These findings provide a better understanding regarding the role of miRNAs in adiposity and insulin sensitivity.
\end{abstract}

\section{Introduction}

The obesity epidemic continues to pose one of the largest worldwide threats to overall health. Obesity is characterised by excessive body fat, occurs in settings of positive energy balances and is strongly associated with insulin resistance, which in turn contributes to a wide variety of chronic diseases states including type 2 diabetes (T2DM), hyperlipidaemia, hypertension and atherosclerosis $(1,2)$.
There is evidence that adipocytes have a central role in the pathogenesis of insulin resistance. Adipocytes can accumulate large amounts of lipid and store it in a manner that is nontoxic to the cells and secrete multiple adipokines that directly regulate whole-body insulin sensitivity. When the capacity of white adipose tissue to store free fatty acid is exceeded, extra-adipose lipid
(ㄷ) 2015 European Society of Endocrinology Printed in Great Britain
Published by Bioscientifica Ltd. 
accumulation, lipotoxicity and insulin resistance ensue (3). Dysregulated adipokine secretion from the expanded adipose tissue of obese individuals also leads to the development of systemic insulin resistance and metabolic diseases (4).

In the past decades, genomic studies have yielded important insights into the pathogenesis of obesity. However, many obese patients remain recalcitrant to medical therapies, and applicable biomarkers for clinical diagnosis are still lacking (5). MicroRNAs (miRNAs) are short non-coding regulatory elements that have an important role in many cellular processes and are promising pharmaceutical targets in various fields, such as cancer and cardiovascular and metabolic diseases $(6,7$, $8,9,10)$. Recent findings have shown that altered circulating miRNAs profiles were linked to pathological conditions, thus opening up the possibility of using miRNAs as promising non-invasive biomarkers for the detection, classification and prognosis of diseases $(11,12)$.

Although basic studies have highlighted the significance of miRNAs in the regulation of obesity and insulin resistance $(13,14,15,16)$, few studies have investigated circulating miRNAs levels as potential biomarkers for obesity in humans. Recently, Ortega et al. (17) have revealed that plasma miR-142-3p, miR-140-5p, miR-15a, miR-520c-3p, and miR-423-5p may constitute novel biomarkers for risk estimation and classification of morbidly obese patients. In a subsequent study, Pescador et al. (18) supported the usage of serum miR-138, miR-15b and miR-376a as valuable predictive biomarkers of obesity. Further clinical evidence is necessary to explore the relevance of circulating miRNAs in maintenance of metabolic homeostasis.

This study sought to investigate the patterns of circulating miRNAs in obese humans and to further explore the independent associations between the concentration of the top-ranked miRNA and parameters of adiposity and insulin sensitivity in young adults. Our findings provide a better understanding regarding the role of miRNAs, particularly miR-122, in obesity and insulin sensitivity.

\section{Subjects and methods}

\section{Ethics statement}

This study protocol was approved by the Institutional Review Board of the Ruijin Hospital, Shanghai Jiao Tong University School of Medicine, and was in accordance with the principle of the Helsinki Declaration II. All of the participants provided written informed consent.

\section{Study population and samples collection}

The detailed characteristics of the 112 individuals included in the discovery study are presented in Supplementary Table 1 , see section on supplementary data given at the end of this article. A total of 230 individuals living in the eastern area of China were recruited for the validation study. One hundred and twenty-three obese patients (BMI $\geq 30 \mathrm{~kg} / \mathrm{m}^{2}$ ) were collected consecutively (from January 2010 to October 2012) from the specialised outpatient clinic for obesity in Ruijin Hospital, Shanghai Jiao Tong University School of Medicine. Patients with syndromatic obesity, diabetes mellitus, hyper- or hypocortisolism, hyper- or hypothyroidism, growth hormone deficiency, severe hepatic or renal disease, acute or chronic infectious or immunological diseases, cancer, pregnancy, and those who were breastfeeding were excluded from our study. One hundred and seven normal weight subjects matching the age and sex distributions of the obese patients were recruited from among volunteers of Shanghai Jiao Tong University School of Medicine on the basis of their normal BMIs $\left(<23 \mathrm{~kg} / \mathrm{m}^{2}\right)$ (19). Normal weight subjects with clinical evidence of major diseases, hypertension, impaired glucose regulation, diabetes mellitus and past history of obesity were excluded from our study. All subjects underwent comprehensive physical examinations and routine biochemical analyses of their blood after an overnight fast. A $75 \mathrm{~g}$ oral glucose tolerance test was also performed. Body fat percentage was quantified using the InBody-720 body composition analyser (Biospace, Seoul, Korea). Clinical and biochemical measurements were assessed as described previously $(20,21)$. Peripheral blood was collected from each participant and was allowed to clot for $1 \mathrm{~h}$ at room temperature. Serum was extracted by centrifugation at $1000 \boldsymbol{g}$ for 15 min at $4{ }^{\circ} \mathrm{C}$, and the supernatant was collected into new tubes and spun at $2000 \mathrm{~g}$ for $10 \mathrm{~min}$ at $4{ }^{\circ} \mathrm{C}$ to remove the few remaining cells. The resultant supernatant was transferred into new tubes and stored at $-80{ }^{\circ} \mathrm{C}$.

\section{Circulating RNA extraction}

We performed RNA extraction using the miRNeasy Mini Kit (Qiagen, Hilden, Germany) according to the manufacturer's instructions. Briefly, $1 \mathrm{ml}$ of QIAzol reagent was added to $200 \mu \mathrm{l}$ of serum sample. The sample was mixed in a tube, followed by the addition of $200 \mu$ l of chloroform. 
After mixing vigorously, the sample was then centrifuged at $12000 \boldsymbol{g}$ for $15 \mathrm{~min}$ at $4{ }^{\circ} \mathrm{C}$. The upper aqueous phase was carefully transferred to a new collection tube, and 1.5 volumes of ethanol were added. The sample was then applied directly to columns and washed. Total RNA was eluted in $30 \mu \mathrm{l}$ of nuclease-free $\mathrm{H}_{2} \mathrm{O}$. The quality and quantity of RNA were evaluated by 260:280 ratio using an Agilent 2100 Bioanalyser (Agilent Technologies, Santa Clara, CA, USA) as described previously (22).

\section{Circulating miRNA microarray}

Serum miRNA samples from 56 obese patients (aged 18-30 years) were randomly divided and combined into two pools (28 samples in each obese pool). Two miRNA pools were similarly obtained from 56 age-, sex- matched normal weight controls (28 samples in each control pool). Genome-wide miRNA expression profiling was performed on Agilent Human miRNA ( $\left.8{ }^{*} 60 \mathrm{~K}\right)$ V16.0 chips (Agilent Technologies) according to the manufacturer's instructions. Briefly, $100 \mathrm{ng}$ of total RNA was dephosphorylated with Calf Intestinal alkaline phosphatase (ALP) at $37^{\circ} \mathrm{C}$ for $30 \mathrm{~min}$ following a denaturalisation with DMSO at $100{ }^{\circ} \mathrm{C}$ for $5-10 \mathrm{~min}$ and a ligation at $16^{\circ} \mathrm{C}$ for $2 \mathrm{~h}$ using miRNA Complete Labelling and Hyb Kit (Agilent Technologies). Labelled RNA was completely dried and resuspended with Blocking Agent and Hybridisation Buffer, incubated at $100{ }^{\circ} \mathrm{C}$ for $5 \mathrm{~min}$ and immediately transferred to an ice water bath for $5 \mathrm{~min}$. The samples were hybridised to the chips (Agilent Technologies) at $55^{\circ} \mathrm{C}$ and 20 r.p.m. for $20 \mathrm{~h}$. Microarrays were then washed with Gene Expression Wash Buffers (Agilent Technologies). They were scanned at $5 \mu \mathrm{m}$ resolution and at 100 and 5\% sensitivity using an Agilent G2565BA Microarray Scanner (Agilent Technologies) under default settings. Images analyses were performed using Agilent Feature Extraction Software 10.7 (Agilent Technologies). Raw data were normalised by Quantile algorithm using Gene Spring Software 11.0 (Agilent Technologies).

\section{Circulating miRNA RT}

We used a fixed volume of $2 \mu \mathrm{l}$ of RNA solution from the $30 \mu \mathrm{l}$ eluate as inputs into each RT reaction using the miRCURY LNA Universal RT Kit (Exiqon, Vedbaek, Denmark) according to the manufacturer's recommendations. A $2 \mu \mathrm{l}$ aliquot of template totol RNA was combined with $2 \mu \mathrm{l}$ of $5 \times$ reaction buffer, $4.5 \mu \mathrm{l}$ of nuclease-free water, $1 \mu l$ of enzyme mix and $0.5 \mu l$ of synthetic spike in to a final volume of $10 \mu \mathrm{l}$. The RT-PCR was set as follows: incubated at $42{ }^{\circ} \mathrm{C}$ for $1 \mathrm{~h}$, heat-inactivated at $95^{\circ} \mathrm{C}$ for $5 \mathrm{~min}$ and immediately cooled to $4{ }^{\circ} \mathrm{C}$ using a Eppendorf mastercycler gradient thermocycler (Eppendorf, Hamburg, Germany). cDNA were diluted to a final volume of $400 \mu$ l.

\section{Analysis of miRNA in individual serum samples by real-time quantitative PCR}

The expression levels of miR-122 in individual serum samples were confirmed with a SYBR-based quantitative PCR using a miR-122-specific primer (Exiqon). The expression levels of miR-122 based on SYBR Green intensity were then assessed by real-time quantitative PCR (qRT-PCR) according to the manufacturer's instructions. A $4 \mu \mathrm{l}$ aliquot of diluted cDNA template was combined with $5 \mu \mathrm{l}$ of SYBR green master mix and $1 \mu \mathrm{l}$ of PCR primer mix to a final volume of $10 \mu$ l. Three technical replicates per sample were used for qRT-PCR amplification run on a LightCycler 480 Real-Time PCR System (Roche Diagnostics). All of the samples were inspected for distinct melting curves and $\mathrm{Tm}$ was checked. The samples must be detected with $C \mathrm{t}<35$ to be included in the analysis.

\section{Definition and calculations}

According to the WHO's diagnostic criteria, we defined subjects with mild, moderate and severe obesity based on BMIs between 30 and $35 \mathrm{~kg} / \mathrm{m}^{2}$, between 35 and $40 \mathrm{~kg} / \mathrm{m}^{2}$, and $\geq 40 \mathrm{~kg} / \mathrm{m}^{2}$ respectively. Homeostasis model assessment of insulin resistance (HOMA-IR) was calculated as (fasting insulin $(\mathrm{IU} / \mathrm{ml}) \times$ fasting glucose $(\mathrm{mmol} / \mathrm{l}) / 22.5)$ (23) Insulin resistance was defined as a HOMA-IR score $>5.268$ (the 75th percentile of HOMA-IR across all subjects).

\section{Statistical analyses}

The data were evaluated for normal distribution with the Shapiro-Wilk test and were logarithmically transformed for statistical analyses when required. Student's $t$-test or ANOVA followed by Bonferroni's multiple comparison analysis was used to examine the differences between the groups of subjects. The miR-122 levels of the different groups were adjusted for comparison by univariate analysis using a general linear model. Pearson's correlations and multiple stepwise regression analysis adjusting for potential confounding factors were performed to examine the associations between miR-122 levels and metabolic parameters. Binary logistic regression was used to predict the risk factors for insulin resistance. 
Table 1 Differential expression of circulating miRNAs in the normal weight and obese groups.

\begin{tabular}{l} 
MicroRNA \\
\hline hsa-miR-636 \\
hsa-miR-933 \\
hsa-miR-122 \\
hsa-miR-574-5p \\
hsa-miR-140-3p \\
hsa-miR-20b \\
hsa-miR-19b \\
hsa-miR-550a \\
hsa-miR-361-5p \\
hsa-miR-17 \\
hsa-miR-30a \\
hsa-miR-654-5p \\
hsa-miR-19a \\
hsa-miR-324-3p \\
hsa-miR-148b \\
hsa-miR-10a \\
hsa-miR-151-3p \\
hsa-miR-335 \\
hsa-miR-126* \\
hsa-miR-142-5p \\
hsa-miR-494 \\
hsa-miR-151-5p \\
hsa-miR-223* \\
hsa-miR-331-3p \\
hsa-miR-144* \\
hsa-miR-199a-5p \\
hsa-miR-195 \\
hsa-miR-301a \\
hsa-miR-338-3p \\
hsa-miR-590-5p \\
hsa-miR-186 \\
hsa-miR-30c \\
hsa-miR-30b \\
hsa-miR-140-5p \\
\hline
\end{tabular}

\begin{tabular}{c}
$\begin{array}{c}\text { Normal weight } \\
\text { group 1 }\end{array}$ \\
\hline 1.3481688 \\
-1.2571797 \\
6.1424985 \\
3.8800778 \\
3.5525482 \\
4.6509943 \\
7.2492414 \\
-1.2637131 \\
1.0862339 \\
4.5317993 \\
1.3116659 \\
-1.3200018 \\
5.9474783 \\
2.6391613 \\
2.2989888 \\
1.0586777 \\
1.0076894 \\
0.26904 \\
2.5720515 \\
3.7417185 \\
1.4936026 \\
2.7396505 \\
0.16948032 \\
-0.47902513 \\
-0.45343864 \\
0.96438444 \\
1.1743264 \\
0.9336167 \\
1.2394677 \\
0.23190765 \\
2.9324102 \\
1.4284668 \\
2.3255095 \\
2.8839657 \\
\hline \\
\end{tabular}

\begin{tabular}{c}
$\begin{array}{c}\text { Normal weight } \\
\text { group } 2\end{array}$ \\
\hline-0.47355455 \\
-0.5452841 \\
6.1424985 \\
3.3068206 \\
3.9628751 \\
5.1532536 \\
7.6516438 \\
-1.1622838 \\
1.1211032 \\
5.107803 \\
2.112866 \\
-1.3200018 \\
6.3565493 \\
2.4187143 \\
2.497092 \\
1.0586777 \\
1.0347425 \\
0.77216923 \\
2.6187317 \\
3.9287333 \\
0.9336167 \\
2.3255095 \\
-1.2571797 \\
0.0879058 \\
0.26904 \\
1.2394677 \\
1.0960096 \\
1.3481688 \\
1.2596201 \\
2.5201914 \\
3.1941247 \\
2.1892483 \\
2.8209538 \\
2.6897788 \\
\hline
\end{tabular}

\begin{tabular}{c}
\hline Obese group 1 \\
\hline 3.3784869 \\
-2.774445 \\
6.7708316 \\
5.2763653 \\
1.3116659 \\
3.4468 \\
5.4974866 \\
-2.8660324 \\
0.16948032 \\
2.8839657 \\
0.77216923 \\
-3.3219576 \\
4.1149077 \\
1.4284668 \\
1.0960096 \\
-0.47355455 \\
-0.628899 \\
-1.2571797 \\
1.0586777 \\
2.2989888 \\
-0.47902513 \\
0.7275481 \\
-3.3219576 \\
-3.3219576 \\
-3.3219576 \\
-3.3219576 \\
-3.3219576 \\
-3.3219576 \\
-3.3219576 \\
-3.3219576 \\
-1.3200018 \\
-3.3219576 \\
-2.8390956 \\
-3.3219576 \\
\hline
\end{tabular}

\begin{tabular}{c}
\hline Obese group 2 \\
\hline 3.2975938 \\
2.1892483 \\
8.4362335 \\
5.1900134 \\
2.6897788 \\
3.0524049 \\
5.8605976 \\
-3.1539028 \\
-3.1539028 \\
3.1317801 \\
-3.1539028 \\
-3.1539028 \\
4.336584 \\
-3.1539028 \\
-3.1539028 \\
-3.1539028 \\
-3.1539028 \\
-3.1539028 \\
-3.1539028 \\
-3.1539028 \\
-3.1539028 \\
-3.1539028 \\
-3.1539028 \\
-3.1539028 \\
-3.1539028 \\
-3.1539028 \\
-3.1539028 \\
-3.1539028 \\
-3.1539028 \\
-3.1539028 \\
-3.1539028 \\
-3.1539028 \\
-3.1539028 \\
-3.1539028 \\
\hline
\end{tabular}

\begin{tabular}{ll}
\hline Fold change \\
\hline 6.194762638 \\
4.264912001 \\
3.224508335 \\
3.056961549 \\
0.326945963 \\
0.316256351 \\
0.292398669 \\
0.289033347 \\
0.287787879 \\
0.280258206 \\
0.267368866 \\
0.265083217 \\
0.261255813 \\
0.242208262 \\
0.212933222 \\
0.199839505 \\
0.186977123 \\
0.182197683 \\
0.181606275 \\
0.175957857 \\
0.175585975 \\
0.151235737 \\
0.137620186 \\
0.11927837 \\
0.109724216 \\
0.049242332 \\
0.048321735 \\
0.047655405 \\
0.044654097 \\
0.030726505 \\
0.030554259 \\
0.029280423 \\
0.020871449 \\
0.015349957 \\
\hline
\end{tabular}

Up/down regulation Up Up Up Down Down Down

Down

Down

Down

Down

Down

Down

Down

Down

Down

Down

Down

Down

Down

Down

Down

Down

Down

Down

Down

Down

Down

Down

Down

Down

Down

Down

Down

The data are presented as quartile normalised fluorescence intensities. Fold change $=$ average (power $(2$, obese group 1$)$, power $(2$, obese group 2$)$ )/average (power (2, normal weight group 1), power (2, normal weight group 2)).

Differences were considered significant for $P<0.05$. All analyses were performed using SAS (version 9.3; SAS Institute, Inc., Cary, NC, USA).

\section{Results}

\section{Comprehensive circulating miRNAs profiling in the discovery samples}

To identify the circulating miRNAs that were involved in the regulation of obesity and insulin sensitivity, we first performed microarray analysis with serum samples from normal weight and obese subjects. We pooled samples within normal weight controls and obese patients, which reduced sample variation and enriched for miRNAs most likely to change between groups. Fold changes $>3$ or below -3 were considered for further analyses. As given in Table 1, 34 circulating miRNAs significantly differed between obese patients and normal weight subjects, including four upregulated miRNAs and 30 suppressed miRNAs in the serum samples of the obese subjects. Among them, miR-122 was the most abundant circulating miRNA. Previous studies have shown that miR-122 is dysregulated in liver metabolic disorders and is involved in cholesterol and lipid regulation $(24,25,26)$. Therefore, the expression of circulating miR-122 was selected for further validation.

\section{Clinical characteristics of the validation population}

The anthropometric and clinical characteristics of the individuals included in the validation study are summarised in Table 2 . There were no significant differences in age or gender between the obese and normal weight 
Table 2 Clinical characteristics of the participants included in the validation study by weight category. The data are presented as the means \pm s.D. or the medians (inter-quartile range).

\begin{tabular}{|c|c|c|c|}
\hline Variable & $\begin{array}{l}\text { Normal weight } \\
\text { controls }(n=107)\end{array}$ & $\begin{array}{c}\text { Obese } \\
\text { patients }(n=123)\end{array}$ & $P$ value \\
\hline \multicolumn{4}{|l|}{ Demographic data } \\
\hline Age (years) & $23.97 \pm 2.88$ & $24.02 \pm 2.61$ & 0.885 \\
\hline Sex, $n(\%)$ & & & 0.580 \\
\hline Male & $50(46.7 \%)$ & $62(50.0 \%)$ & \\
\hline Female & $57(53.3 \%)$ & $61(50.0 \%)$ & \\
\hline \multicolumn{4}{|l|}{ Anthropometric measurements } \\
\hline BMI $\left(\mathrm{kg} / \mathrm{m}^{2}\right)$ & $20.79 \pm 1.41$ & $37.73 \pm 4.40$ & $<0.001$ \\
\hline Waist-to-hip ratio & $0.80 \pm 0.05$ & $0.98 \pm 0.09$ & $<0.001$ \\
\hline Fat percentage $(\%)$ & $22.27 \pm 4.52$ & $43.56 \pm 6.99$ & $<0.001$ \\
\hline Systolic blood pressure $(\mathrm{mmHg})$ & $112.81 \pm 14.64$ & $124.56 \pm 13.96$ & $<0.001$ \\
\hline Diastolic blood pressure $(\mathrm{mmHg})$ & $70.25 \pm 9.68$ & $83.19 \pm 10.71$ & $<0.001$ \\
\hline \multicolumn{4}{|l|}{ Biochemical characteristics } \\
\hline Alanine aminotransferase (IU/I) & $15.00(13.00-19.00)$ & $44.00(28.00-77.00)$ & $<0.001$ \\
\hline Aspartate aminotransferase (IU/I) & $19.00(17.00-22.00)$ & $27.00(20.00-40.00)$ & $<0.001$ \\
\hline Alkaline phosphatase (IU/I) & $56.50(48.00-71.00)$ & $63.50(51.00-74.00)$ & 0.049 \\
\hline Gamma glutamyl transferase (IU/I) & $13.00(11.00-16.00)$ & $31.00(18.00-51.00)$ & $<0.001$ \\
\hline Triglycerides $(\mathrm{mmol} / \mathrm{l})$ & $0.83(0.61-1.03)$ & $1.46(1.05-1.90)$ & $<0.001$ \\
\hline Total cholesterol (mmol/l) & $4.05(3.73-4.56)$ & $4.36(3.90-4.95)$ & 0.866 \\
\hline HDL-cholesterol (mmol/l) & $1.56(1.36-1.73)$ & $1.04(0.92-1.23)$ & $<0.001$ \\
\hline LDL-cholesterol $(\mathrm{mmol} / \mathrm{l})$ & $2.29(1.95-2.63)$ & $2.76(2.35-3.18)$ & $<0.001$ \\
\hline Fasting blood glucose (mmol/l) & $4.68 \pm 0.40$ & $5.23 \pm 0.89$ & $<0.001$ \\
\hline 2-h blood glucose $(\mathrm{mmol} / \mathrm{l})$ & $5.14 \pm 0.98$ & $6.81 \pm 2.04$ & $<0.001$ \\
\hline Fasting insulin $(\mu \mathrm{IU} / \mathrm{ml})$ & $6.81(4.91-8.83)$ & $21.49(15.34-28.76)$ & $<0.001$ \\
\hline $2-\mathrm{h}$ insulin $(\mu \mathrm{IU} / \mathrm{ml})$ & $33.92(18.57-52.37)$ & $117.90(72.05-178.30)$ & $<0.001$ \\
\hline \multicolumn{4}{|l|}{ Insulin resistance } \\
\hline HOMA-IR $\left(\mu \mathrm{IU} \cdot \mathrm{mol} / \mathrm{I}^{2}\right)$ & $1.41(1.01-1.81)$ & $5.11(3.63-6.48)$ & $<0.001$ \\
\hline
\end{tabular}

groups (Table 2). As expected, BMI, waist-to-hip ratio (WHR), fat percentage, blood pressure, hepatic enzymes, triglycerides (TG), LDL-cholesterol (LDL-c), fasting glucose and insulin, 2-h glucose, 2-h insulin and HOMA-IR were significantly higher in the obese subjects. The obese participants also had significantly lower levels of HDLcholesterol (HDL-c) than did the normal weight subjects. However, total cholesterol (TC) was not significantly different between the groups (Table 2).

\section{Validation of miR-122 expression in individual serum samples}

To validate the possibility of using miR-122 as a biomarker for obesity, serum miR-122 levels were individually determined by qRT-PCR. Consistent with the microarray data, we found that the miR-122 relative expression levels were markedly higher in the obese subjects, with a 3.07-fold increase, than in the normal weight subjects $\left(P=7.10 \times 10^{-12}\right.$; Fig. $\left.1 \mathrm{~A}\right)$. The means \pm s.D. of $C t$ values were $32.37 \pm 1.55$ and $30.85 \pm 1.77$ in normal weight and obese subjects respectively (Fig. 1B). Indeed, miR-122 expression levels remained significantly elevated in obese patients after adjusting for confounding factors such as age, sex, hepatic enzymes and HDL-c $(P<0.001$; Fig. 1C).

To further explore the relationship between miR-122 and obesity, we stratified the degree of obesity by BMI. Compared with the normal weight subjects, the mildly, moderately and severely obese patients all had significantly higher levels of circulating miR-122 (all $P<0.05$; Fig. 2A). Circulating miR-122 exhibited a tendency to increase with the degree of obesity, although this pattern was not significant. After adjusting for age, sex, alanine aminotransferase (ALT) and HDL-c, miR-122 expression remained significantly different between the normal weight and obese groups (all $P<0.01$; Fig. 2B).

\section{Correlates of serum miR-122 relative expression levels}

miR-122 levels were significantly correlated with sex, BMI, WHR, fat percentage, blood pressure, ALT, aspartate aminotransferase (AST), ALP, gamma glutamyl transferase (GGT), TG, HDL-c, fasting blood glucose, 2-h blood glucose, fasting insulin, 2-h insulin and HOMA-IR (all $P<0.01$; Table 3). Multiple stepwise regression analysis demonstrated an independent association of miR-122 with ALT $(\beta=0.648, P<0.001$; Table 3$)$. 

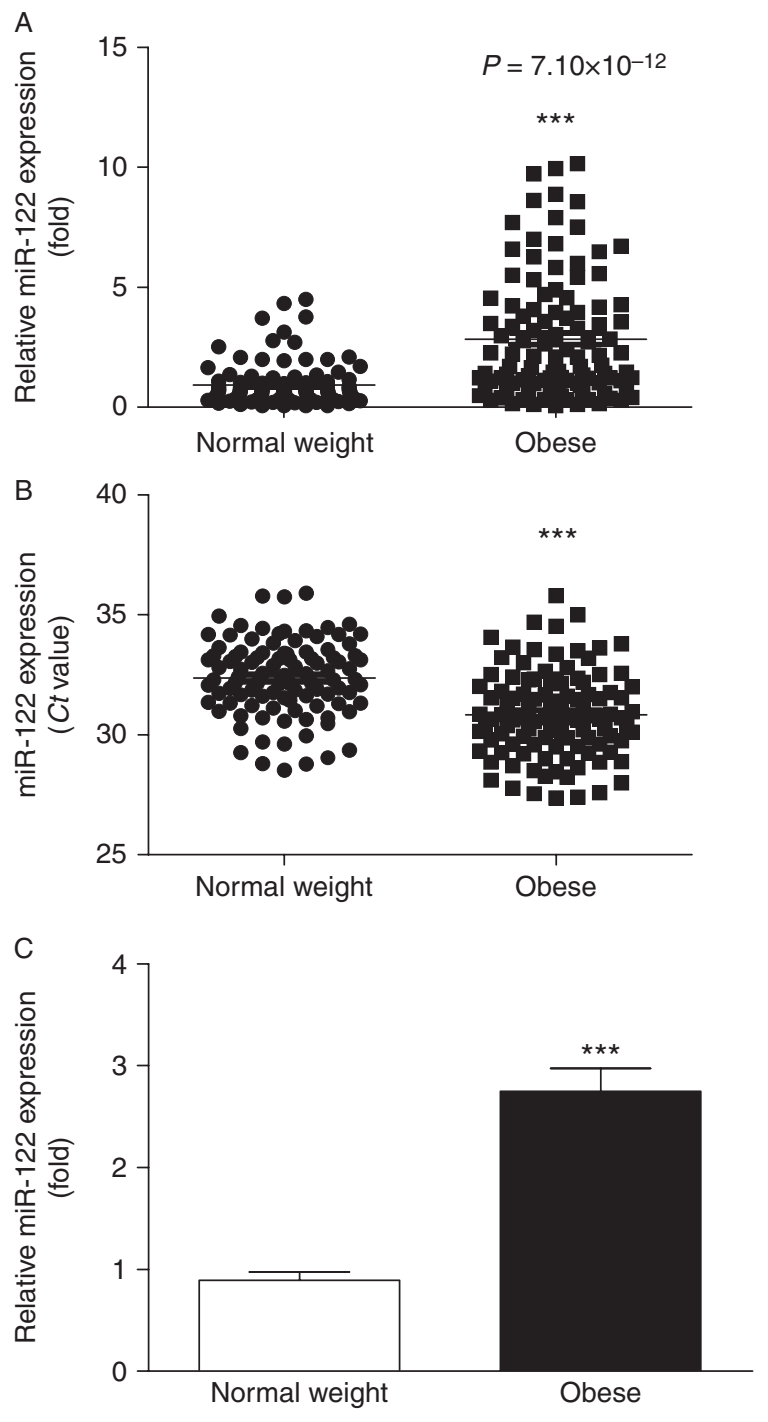

\section{Figure 1}

The circulating miR-122 levels were elevated in the obese subjects compared with the normal weight controls.

(A) Differential relative miR-122 expression levels verified by qRT-PCR in the serum samples from normal weight $(n=107)$ and obese ( $n=123$ ) subjects. (B) Differential Ct values of miR-122 expression verified by qRT-PCR in the serum samples from normal weight and obese subjects. (C) After adjusting for age, sex, ALT and HDL-C, miR-122 expression remained significantly different between the normal weight and obese participants. The mean values are plotted with the S.E.M.S. $* * * P<0.001$ vs normal weight control group.

Given that the insulin sensitivity, glucose regulation and liver function of normal weight and obese subjects might vary substantially, we further analysed the correlations between miR-122 relative expression

levels and covariables separately in the normal weight and obese subjects. In the normal weight subjects, miR-122 expression was significantly associated with sex, ALT, ALP, GGT, HDL-c, blood glucose and fasting insulin. Multiple stepwise regression analysis revealed
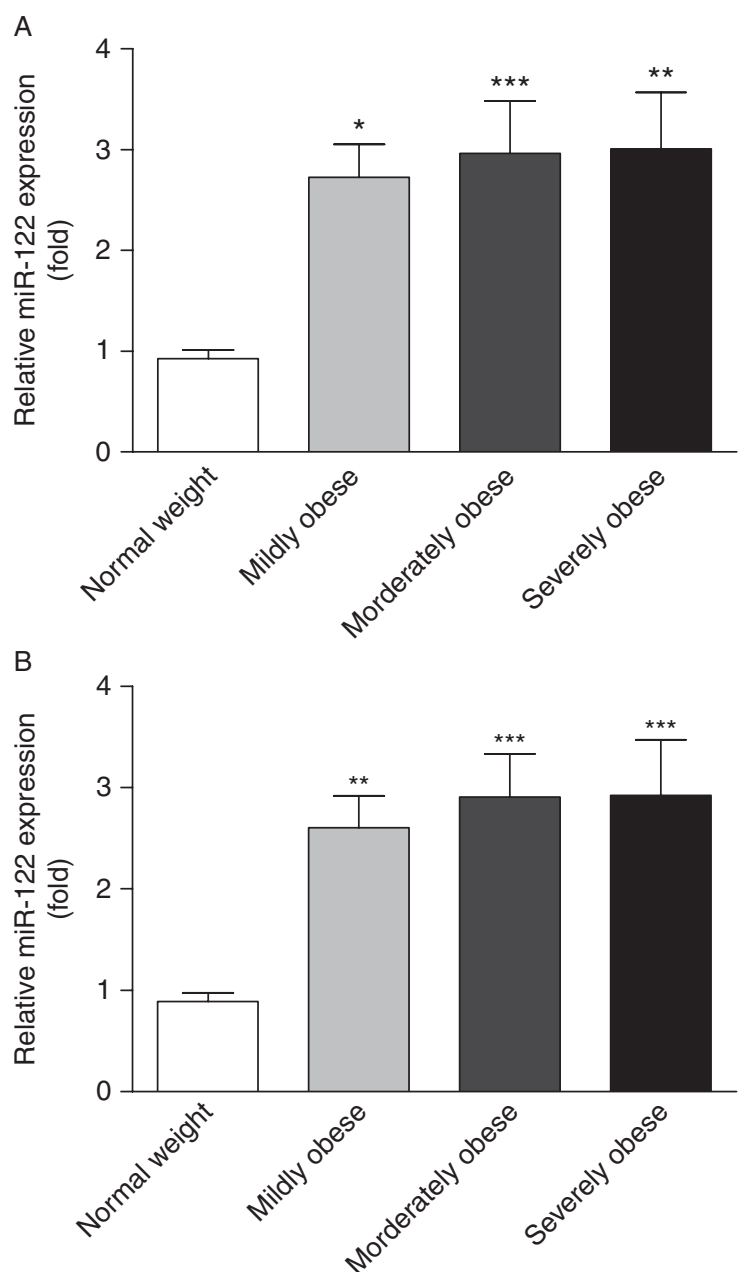

\section{Figure 2}

Differential miR-122 expression in the serum samples of patients with different degrees of obesity compared with the normal weight controls. (A) Mildly $(n=28)$, moderately $(n=66)$ and severely $(n=29)$ obese patients all had significantly higher levels of circulating miR-122 than did the normal weight ( $n=107$ ) subjects, but the miR-122 expressions tended to be unchanged as the degree of obesity increased. (B) After adjusting for age, sex, ALT and HDL-C, miR-122 expression remained significantly different between the normal weight and obese subjects. The mean values are plotted with the S.E.M.S. $* P<0.05, * * P<0.01$ and $* * * P<0.001$ vs the normal weight control group. 
Table 3 Pearson's correlation and stepwise regression analyses of miR-122 with indices of adiposity and metabolic risk.

\begin{tabular}{|c|c|c|c|c|}
\hline \multirow[b]{2}{*}{ Covariables } & \multicolumn{2}{|c|}{ Pearson's correlation } & \multicolumn{2}{|c|}{ Stepwise } \\
\hline & $r$ & $P$ value & $\beta$ & $P$ value \\
\hline \multicolumn{5}{|l|}{ Demographic data } \\
\hline Age (years) & 0.042 & 0.531 & 0.035 & 0.653 \\
\hline Sex & -0.187 & 0.004 & -0.063 & 0.426 \\
\hline \multicolumn{5}{|l|}{ Anthropometric measurements } \\
\hline BMI $\left(\mathrm{kg} / \mathrm{m}^{2}\right)$ & 0.469 & $<0.001$ & 0.035 & 0.656 \\
\hline Waist-to-hip ratio & 0.383 & $<0.001$ & - & - \\
\hline Fat percentage $(\%)$ & 0.400 & $<0.001$ & - & - \\
\hline Systolic blood pressure (mmHg) & 0.260 & $<0.001$ & 0.048 & 0.545 \\
\hline Diastolic blood pressure $(\mathrm{mmHg})$ & 0.287 & $<0.001$ & 0.061 & 0.437 \\
\hline \multicolumn{5}{|l|}{ Biochemical characteristics } \\
\hline Alanine aminotransferase (IU/I) & 0.634 & $<0.001$ & 0.648 & $<0.001$ \\
\hline Aspartate aminotransferase (IU/I) & 0.575 & $<0.001$ & - & - \\
\hline Alkaline phosphatase (IU/I) & 0.257 & 0.001 & - & - \\
\hline Gamma glutamyl transferase (IU/I) & 0.532 & $<0.001$ & - & - \\
\hline Triglycerides $(\mathrm{mmol} / \mathrm{l})$ & 0.448 & $<0.001$ & 0.047 & 0.555 \\
\hline Total cholesterol (mmol/l) & 0.033 & 0.641 & - & - \\
\hline HDL-cholesterol (mmol/l) & -0.351 & $<0.001$ & -0.039 & 0.617 \\
\hline LDL-cholesterol (mmol/l) & 0.143 & 0.054 & - & - \\
\hline Fasting blood glucose (mmol/l) & 0.345 & $<0.001$ & - & - \\
\hline $2 \mathrm{~h}$ blood glucose $(\mathrm{mmol} / \mathrm{l})$ & 0.249 & $<0.001$ & - & - \\
\hline Fasting insulin $(\mu \mathrm{IU} / \mathrm{ml})$ & 0.437 & $<0.001$ & - & - \\
\hline $2 \mathrm{~h}$ insulin $(\mu \mathrm{IU} / \mathrm{ml})$ & 0.315 & $<0.001$ & - & - \\
\hline \multicolumn{5}{|l|}{ Insulin resistance } \\
\hline HOMA-IR $\left(\mu \mathrm{IU} \cdot \mathrm{mol} / \mathrm{I}^{2}\right)$ & 0.401 & $<0.001$ & 0.097 & 0.217 \\
\hline
\end{tabular}

$r$, Pearson's correlation coefficient; $\beta$, standardised regression coefficient; ' - ', indicates that the variable was not included in the multiple stepwise regression modelling.

independent associations of miR-122 with sex $(\beta=-0.425, P<0.001)$ and HDL-c $(\beta=-0.287, P=0.017$; Supplementary Table 2 , see section on supplementary data given at the end of this article). In obese patients, miR-122 expression was significantly associated with biochemical characteristics that included ALT, AST, GGT, TG, fasting and $2 \mathrm{~h}$ blood glucose, and was independently associated with $\operatorname{ALT}(\beta=0.554, P<0.001$; Supplementary Table 3).

\section{Association of miR-122 with insulin resistance}

In the univariate model, in addition to miR-122, multiple parameters were found to be significantly related to
HOMA-IR, including BMI, WHR, fat percentage, systolic blood pressure (SBP), diastolic blood pressure (DBP), ALT, HDL-c, LDL-c, fasting blood glucose and fasting insulin (all $P<0.01$, data not shown). We next examined the relationship between circulating miR-122 levels and insulin resistance in our study population. Binary logistic regression was performed to analyse the independent risk factors for insulin resistance. Each s.D. unit increase in miR-122 level was associated with a 331.9\% greater odds of insulin resistance in a model adjusted for age and gender. After further adjusting for obesity, blood pressure, lipid profile and liver function, miR-122 remained positively associated with an elevated likelihood of insulin resistance $(\mathrm{OR}=3.379 /$ s.D. unit, 95\% CI=1.141-10.007, $P=0.028$; Table 4).

Table 4 The multiple stepwise regression modelling for binary insulin resistance associated with per unit increases in S.D. in the serum miR-122 levels.

\begin{tabular}{|c|c|c|c|}
\hline \multirow[b]{2}{*}{ Model } & \multirow[b]{2}{*}{ Adjustment } & \multicolumn{2}{|c|}{ Binary insulin resistance ( $>75$ th percentile vs lower) } \\
\hline & & OR $(95 \% \mathrm{Cl})$ & $P$ value \\
\hline Model 1 & Adjusted for age and sex & $4.319(2.383-7.826)$ & $<0.001$ \\
\hline Model 2 & $\begin{array}{l}\text { Further adjusted for BMI, WHR, fat percentage, SBP, } \\
\text { DBP, lipid profile and liver function based on model } 1\end{array}$ & $3.379(1.141-10.007)$ & 0.028 \\
\hline
\end{tabular}

OR, odds ratio; WHR, waist-to-hip ratio; SBP, systolic blood pressure; DBP, diastolic blood pressure. 


\section{Discussion}

Previous literature has unequivocally demonstrated that endogenous serum and plasma miRNAs are sufficiently stable and quantifiable to serve as clinical biomarkers $(11,12)$. Considering that clinical specimens of serum are more plentiful than plasma samples in many clinical sample repositories, it is essential to establish whether miRNAs levels are different in serum compared with plasma. In this regard, Mitchell et al. (11) found that the abundance of miRNAs in serum and plasma were strongly correlated, which indicates that both serum and plasma samples would be suitable for investigations of miRNAs as blood-based biomarkers.

Mammalian circulating miRNAs are packaged inside lipid or lipidprotein complexes, such as microvesicles, exosomes or apoptotic bodies $(27,28,29)$. Studies have shown that associations with proteins $(30,31)$ also make miRNAs resistant to ribonuclease activity. miRNAs secreted inside exosomes may have a different fate compared with those associated with proteins or HDL $(32,33)$. Specific miRNAs tend to be preferentially released by cells and are thus more prone to be found in circulation (34); however, changes in the levels of circulating miRNAs do not necessarily reflect deregulation of miRNAs expression inside cells (22). Therefore, the use of miRNAs as biomarkers should not be confounded with their regulatory functions inside the cells. Although emerging evidence has established that extracellular miRNAs contained in the body fluids have distinct physiological function $(11,12,35)$, the main function and the meaning of the existence of circulating miRNAs remain elusive.

The role of miRNAs in diabetes and obesity has recently been studied. Ortega et al. (17) established the foundation to motivate further global investigations of miRNAs as circulating biomarkers for obesity in humans. They suggested that plasma miR-142-3p, miR-140-5p, miR-15a, miR-520c-3p and miR-423-5p may constitute novel biomarkers for risk estimation and classification of morbidly obese patients. By contrast, another study demonstrated that miR-138 or miR-376a provided a powerful predictive tool for distinguishing patients with obesity from healthy controls, patients with T2DM and obese diabetic patients, whereas a combination of miR-138 and miR-503 can distinguish diabetic from obese diabetic patients (18). This might be owing to the different clinical characteristics of these studies and the lack of a generally accepted endogenous miRNA control to normalise for circulating miRNAs levels. In addition, a different extraction and quantification method among the studies also contribute to the conflicting results. Future studies are warranted for the identification of an accurate normalisation protocol and the empirical validation of stable endogenous control miRNAs.

Ortega et al. (17) found that plasma miR-122 concentration increased in moderately obese patients but decreased in morbidly obese patients. Intriguingly, circulating miR-122 concentrations significantly decreased after surgery-induced weight loss (17). By contrast, our data demonstrated that circulating miR-122 was robustly elevated in the obese patients and exhibited a tendency to increase with the degree of obesity. Moreover, we observed a significantly direct association between serum miR-122 levels and BMI in our cohort consisting of a total of 230 Chinese young adults, although Ortega et al. (17) reported an inverse correlation between circulating miR-122 concentrations and BMI in white men. It is likely that this disparity is partly related to the variance in the definition of obesity, comparably healthier glucose metabolic state (only 23 obese patients had impaired glucose regulation), a much younger age and a better blood pressure in our study population.

The first miRNA linked to metabolic control, miR-122, is primarily expressed in the liver and is initially shown to affect hepatic cholesterol and fatty-acid metabolism in mice $(24,25)$. In a subsequent study, miR-122 was targeted by antisense inhibitors in African green monkeys, resulting in dose-dependent lowering of plasma cholesterol (26). By contrast, we noted no correlation between miR122 and TC in humans. Indeed, our data suggested that miR-122 concentration was significantly correlated with TG and HDL-c in all participants. Likewise, an independent association of miR-122 with HDL-c was determined in the lean healthy subjects. This might be due, in part, to the characteristics of our study population. Large studies regarding circulating miRNAs levels from individuals of both genders and various ages will be worthwhile.

In line with the results of animal studies, we found miR-122 expression was significantly associated with hepatic enzymes in humans. Moreover, we found an independent association of miR-122 with ALT, as demonstrated by multiple stepwise regression analysis. Wang et al. (22) reported that mouse plasma miR-122 exhibited dose- and exposure duration-dependent changes that parallel aminotransferase levels and liver degeneration and might be more sensitive and reliable than ALT levels. Further investigations should identify whether the sensitivity and reliability of circulating miR-122 are better than those of ALT in humans to confirm its clinical applicability. 
In summary, this study provides clinical evidence revealing that circulating miRNAs are dysregulated in human obesity. Apart from detecting the involvement of aberrant circulating miR-122 under an obese state, we found that circulating miR-122 levels were closely correlated with glucose regulation and insulin sensitivity in Chinese young adults. Most importantly, we demonstrated that miR-122 was positively associated with increased odds of insulin resistance in humans, indicating its potential diagnostic value. Our findings suggest that circulating miR-122 may act as a promising biomarker of obesity and insulin resistance.

Supplementary data

This is linked to the online version of the paper at http://dx.doi.org/10.1530/ EJE-14-0867.

\section{Declaration of interest}

The authors declare that there is no conflict of interest that could be perceived as prejudicing the impartiality of the research reported.

\section{Funding}

This work was supported by grants from the National Nature Science Foundation of China (nos 81270931, 81471060, 81170739, 81130016, 81390350 and 81270859$)$

\section{Author contribution statement}

R Wang, Y Cao and J Hong performed the experiments; G Ning, W Wang and J Hong conceived and designed all the experiments; R Wang, J Shi, W Gu, Y Zhang, W Wang and G Ning analysed the data; R Wang, Y Cao, $J$ Hong and J Shi wrote the manuscript; and all authors agreed on the final manuscript.

\section{Acknowledgements}

The authors thank Qun Yan, Ying Zhai, Yingchao Chen, Li Wang and Lin Miao (Department of Endocrinology and Metabolism, Ruijin Hospital, Shanghai Jiao Tong University School of Medicine) for assistance in collecting serum samples from subjects.

\section{References}

1 Lean ME, Han TS \& Seidell JC. Impairment of health and quality of life in people with large waist circumference. Lancet $1998 \mathbf{3 5 1} 853-856$. (doi:10.1016/S0140-6736(97)10004-6)

2 Ogden CL, Carroll MD, Kit BK \& Flegal KM. Prevalence of obesity and trends in body mass index among US children and adolescents, 1999-2010. Journal of the American Medical Association 2012307 483-490. (doi:10.1001/jama.2012.40)
3 Savage DB, Petersen KF \& Shulman GI. Mechanisms of insulin resistance in humans and possible links with inflammation. Hypertension 200545 828-833. (doi:10.1161/01.HYP.0000163475.04421.e4)

4 Rosen ED \& Spiegelman BM. Adipocytes as regulators of energy balance and glucose homeostasis. Nature 2006444 847-853. (doi:10.1038/ nature05483)

5 Bray GA \& Tartaglia LA. Medicinal strategies in the treatment of obesity. Nature 2000404 672-677. (doi:10.1038/35007544)

6 Couzin J. MicroRNAs make big impression in disease after disease. Science 2008319 1782-1784. (doi:10.1126/science.319.5871.1782)

7 D'Alessandra Y, Devanna P, Limana F, Straino S, Di Carlo A, Brambilla PG, Rubino M, Carena MC, Spazzafumo L, De Simone M et al. Circulating microRNAs are new and sensitive biomarkers of myocardial infarction. European Heart Journal 201031 2765-2773. (doi:10.1093/ eurheartj/ehq167)

8 Lu J, Getz G, Miska EA, Alvarez-Saavedra E, Lamb J, Peck D, SweetCordero A, Ebert BL, Mak RH, Ferrando AA et al. MicroRNA expression profiles classify human cancers. Nature 2005435 834-838. (doi:10. 1038/nature03702)

9 Zampetaki A, Kiechl S, Drozdov I, Willeit P, Mayr U, Prokopi M, Mayr A Weger S, Oberhollenzer F, Bonora E et al. Plasma microRNA profiling reveals loss of endothelial miR-126 and other microRNAs in type 2 diabetes. Circulation Research 2010107 810-817. (doi:10.1161/CIRCRESAHA.110.226357)

10 Karolina DS, Tavintharan S, Armugam A, Sepramaniam S, Pek SL, Wong MT, Lim SC, Sum CF \& Jeyaseelan K. Circulating miRNA profiles in patients with metabolic syndrome. Journal of Clinical Endocrinology and Metabolism 201297 E2271-E2276. (doi:10.1210/jc.2012-1996)

11 Mitchell PS, Parkin RK, Kroh EM, Fritz BR, Wyman SK, PogosovaAgadjanyan EL, Peterson A, Noteboom J, O'Briant KC, Allen A et al. Circulating microRNAs as stable blood-based markers for cancer detection. PNAS 2008105 10513-10518. (doi:10.1073/pnas. 0804549105)

12 Chen X, Ba Y, Ma L, Cai X, Yin Y, Wang K, Guo J, Zhang Y, Chen J, Guo X et al. Characterization of microRNAs in serum: a novel class of biomarkers for diagnosis of cancer and other diseases. Cell Research 200818 997-1006. (doi:10.1038/cr.2008.282)

13 Xie H, Lim B \& Lodish HF. MicroRNAs induced during adipogenesis that accelerate fat cell development are downregulated in obesity. Diabetes 200958 1050-1057. (doi:10.2337/db08-1299)

$14 \mathrm{Xu}$ P, Vernooy SY, Guo M \& Hay BA. The Drosophila microRNA mir-14 suppresses cell death and is required for normal fat metabolism. Current Biology 200313 790-795. (doi:10.1016/S0960-9822(03)00250-1)

15 Teleman AA, Maitra S \& Cohen SM. Drosophila lacking microRNA miR278 are defective in energy homeostasis. Genes and Development 200620 417-422. (doi:10.1101/gad.374406)

16 Trajkovski M, Hausser J, Soutschek J, Bhat B, Akin A, Zavolan M, Heim MH \& Stoffel M. MicroRNAs 103 and 107 regulate insulin sensitivity. Nature 2011474 649-653. (doi:10.1038/nature10112)

17 Ortega FJ, Mercader JM, Catalan V, Moreno-Navarrete JM, Pueyo N, Sabater M, Gomez-Ambrosi J, Anglada R, Fernandez-Formoso JA, Ricart W et al. Targeting the circulating microRNA signature of obesity. Clinical Chemistry 201359 781-792. (doi:10.1373/clinchem.2012. 195776)

18 Pescador N, Perez-Barba M, Ibarra JM, Corbaton A, Martinez-Larrad MT $\&$ Serrano-Rios M. Serum circulating microRNA profiling for identification of potential type 2 diabetes and obesity biomarkers. PLOS ONE 20138 e77251. (doi:10.1371/journal.pone.0077251)

19 Consulation WE. Appropriate body-mass index for Asian populations and its implications for policy and intervention strategies. Lancet 2004 363 157-163. (doi:10.1016/S0140-6736(03)15268-3)

20 Shi J, Zhang Y, Gu W, Cui B, Xu M, Yan Q, Wang W, Ning G \& Hong J. Serum liver fatty acid binding protein levels correlate positively with obesity and insulin resistance in Chinese young adults. PLOS ONE 2012 7 e48777. (doi:10.1371/journal.pone.0048777) 
21 Wang R, Hong J, Liu R, Chen M, Xu M, Gu W, Zhang Y, Ma Q, Wang F, Shi $\mathrm{J}$ et al. SFRP5 acts as a mature adipocyte marker but not as a regulator in adipogenesis. Journal of Molecular Endocrinology 201453 405-415. (doi:10.1530/JME-14-0037)

22 Wang K, Zhang S, Marzolf B, Troisch P, Brightman A, Hu Z, Hood LE \& Galas DJ. Circulating microRNAs, potential biomarkers for druginduced liver injury. PNAS 2009106 4402-4407. (doi:10.1073/pnas. 0813371106)

23 Matthews DR, Hosker JP, Rudenski AS, Naylor BA, Treacher DF \& Turner RC. Homeostasis model assessment: insulin resistance and $\beta$-cell function from fasting plasma glucose and insulin concentrations in man. Diabetologia 198528 412-419. (doi:10.1007/BF00280883)

24 Esau C, Davis S, Murray SF, Yu XX, Pandey SK, Pear M, Watts L, Booten SL, Graham M, McKay R et al. miR-122 regulation of lipid metabolism revealed by in vivo antisense targeting. Cell Metabolism 20063 87-98. (doi:10.1016/j.cmet.2006.01.005)

25 Krutzfeldt J, Rajewsky N, Braich R, Rajeev KG, Tuschl T, Manoharan M $\&$ Stoffel M. Silencing of microRNAs in vivo with 'antagomirs'. Nature 2005438 685-689. (doi:10.1038/nature04303)

26 Elmen J, Lindow M, Schutz S, Lawrence M, Petri A, Obad S, Lindholm M, Hedtjarn M, Hansen HF, Berger U et al. LNA-mediated microRNA silencing in non-human primates. Nature $2008 \mathbf{4 5 2}$ 896-899. (doi:10.1038/nature06783)

27 Valadi H, Ekstrom K, Bossios A, Sjostrand M, Lee JJ \& Lotvall JO. Exosome-mediated transfer of mRNAs and microRNAs is a novel mechanism of genetic exchange between cells. Nature Cell Biology 2007 9 654-659. (doi:10.1038/ncb1596)

28 Zernecke A, Bidzhekov K, Noels H, Shagdarsuren E, Gan L, Denecke B, Hristov M, Koppel T, Jahantigh MN, Lutgens E et al. Delivery of
microRNA-126 by apoptotic bodies induces CXCL12-dependent vascular protection. Science Signaling 20092 ra81. (doi:10.1126/ scisignal.2000610)

29 Hunter MP, Ismail N, Zhang X, Aguda BD, Lee EJ, Yu L, Xiao T, Schafer J, Lee ML, Schmittgen TD et al. Detection of microRNA expression in human peripheral blood microvesicles. PLoS ONE 20083 e3694. (doi:10.1371/journal.pone.0003694)

30 Turchinovich A, Weiz L, Langheinz A \& Burwinkel B. Characterization of extracellular circulating microRNA. Nucleic Acids Research 201139 7223-7233. (doi:10.1093/nar/gkr254)

31 Wang K, Zhang S, Weber J, Baxter D \& Galas DJ. Export of microRNAs and microRNA-protective protein by mammalian cells. Nucleic Acids Research 201038 7248-7259. (doi:10.1093/nar/gkq601)

32 Vickers KC, Palmisano BT, Shoucri BM, Shamburek RD \& Remaley AT. MicroRNAs are transported in plasma and delivered to recipient cells by high-density lipoproteins. Nature Cell Biology 201113 423-433. (doi:10.1038/ncb2210)

33 Eichelser C, Stuckrath I, Muller V, Milde-Langosch K, Wikman H, Pantel K \& Schwarzenbach H. Increased serum levels of circulating exosomal microRNA-373 in receptor-negative breast cancer patients. Oncotarget 20145 9650-9663.

34 Guduric-Fuchs J, O'Connor A, Camp B, O'Neill CL, Medina RJ \& Simpson DA. Selective extracellular vesicle-mediated export of an overlapping set of microRNAs from multiple cell types. BMC Genomics 201213 357. (doi:10.1186/1471-2164-13-357)

35 Gilad S, Meiri E, Yogev Y, Benjamin S, Lebanony D, Yerushalmi N, Benjamin H, Kushnir M, Cholakh H, Melamed N et al. Serum microRNAs are promising novel biomarkers. PLoS ONE 20083 e3148. (doi:10.1371/journal.pone.0003148)

Received 12 October 2014

Revised version received 11 December 2014

Accepted 16 December 2014 